\title{
Effects of fast rotation on the wind of Luminous Blue Variables
}

\author{
Jose H. Groh \\ Max-Planck-Institut für Radioastronomie, Auf dem Hügel 69, D-53121 Bonn, Germany \\ email: jgroh@mpifr.de
}

\begin{abstract}
While theoretical studies have long suggested a fast-rotating nature of Luminous Blue Variables (LBVs), observational confirmation of fast rotation was not detected until recently. Here I discuss the diagnostics that have allowed us to constrain the rotational velocity of LBVs: broadening of spectral lines and latitude-dependent variations of the wind density structure. While rotational broadening can be directly detected using high-resolution spectroscopy, longbaseline near-infrared interferometry is needed to directly measure the shape of the latitudedependent photosphere that forms in a fast-rotating star. In addition, complex 2-D radiative transfer models need to be employed if one's goal is to constrain rotational velocities of LBVs. Here I illustrate how the above methods were able to constrain the rotational velocities of the LBVs AG Carinae, HR Carinae, and Eta Carinae.
\end{abstract}

Keywords. stars: rotation, stars: atmospheres, stars: emission-line, techniques: interferometric

\section{Introduction}

Tremendous advancement on understanding the evolution of massive stars has been achieved in the last decades (e. g., Maeder \& Meynet 1994), in particular after the inclusion of the effects of rotation in evolutionary models (Maeder \& Meynet 2000b). These models predict the existence of a short-lived, transitional stage, usually referred to as the Luminous Blue Variable (LBV) phase (Conti 1984), when the star has a high massloss rate $\left(\dot{M} \sim 10^{-5}-10^{-3} M_{\odot} \mathrm{yr}^{-1}\right)$. In the current picture of stellar evolution, LBVs are rapidly evolving massive stars in the transitory phase from being an O-type star to becoming a Wolf-Rayet star (Humphreys \& Davidson 1994; Meynet \& Maeder 2003).

Although the effects of rotation on classical O-type stars have been documented for almost a century, their more active descendants, the Luminous Blue Variables, have eluded direct detection of fast rotation until recently, when the prototype LBV AG Carinae was found to be a fast rotator (Groh et al. 2006). Obtaining the rotational velocity $\left(v_{\text {rot }}\right)$ of LBVs is challenging because of the presence of (a) an optically-thick wind caused by the large mass-loss rate of the star and (b) time variability of the stellar radius due to the $\mathrm{S}$-Dor type variability.

The presence of a dense wind causes the formation of an extended photosphere, making the use of a radiative transfer code mandatory to obtain information about the hydrostatic core. Time variability caused by the S-Dor cycles, characterized by changes of up to a factor of 10 in the hydrostatic radius of LBVs, imply variations of $v_{\text {rot }}$ as a function of time. The issue is that LBVs are apparently closer to the critical rotational velocity for break-up ( $v_{\text {crit }}$ ) only during visual minimum epochs of the S-Dor cycle (Groh et al. 2006; Groh et al. 2010, in prep.). Unfortunately, LBVs spend most of their time at visual maximum, requiring continuous spectroscopic and photometric monitoring (and a great deal of patience!) to catch the star at the right time and obtain their maximum value of $v_{\text {rot }}$. 


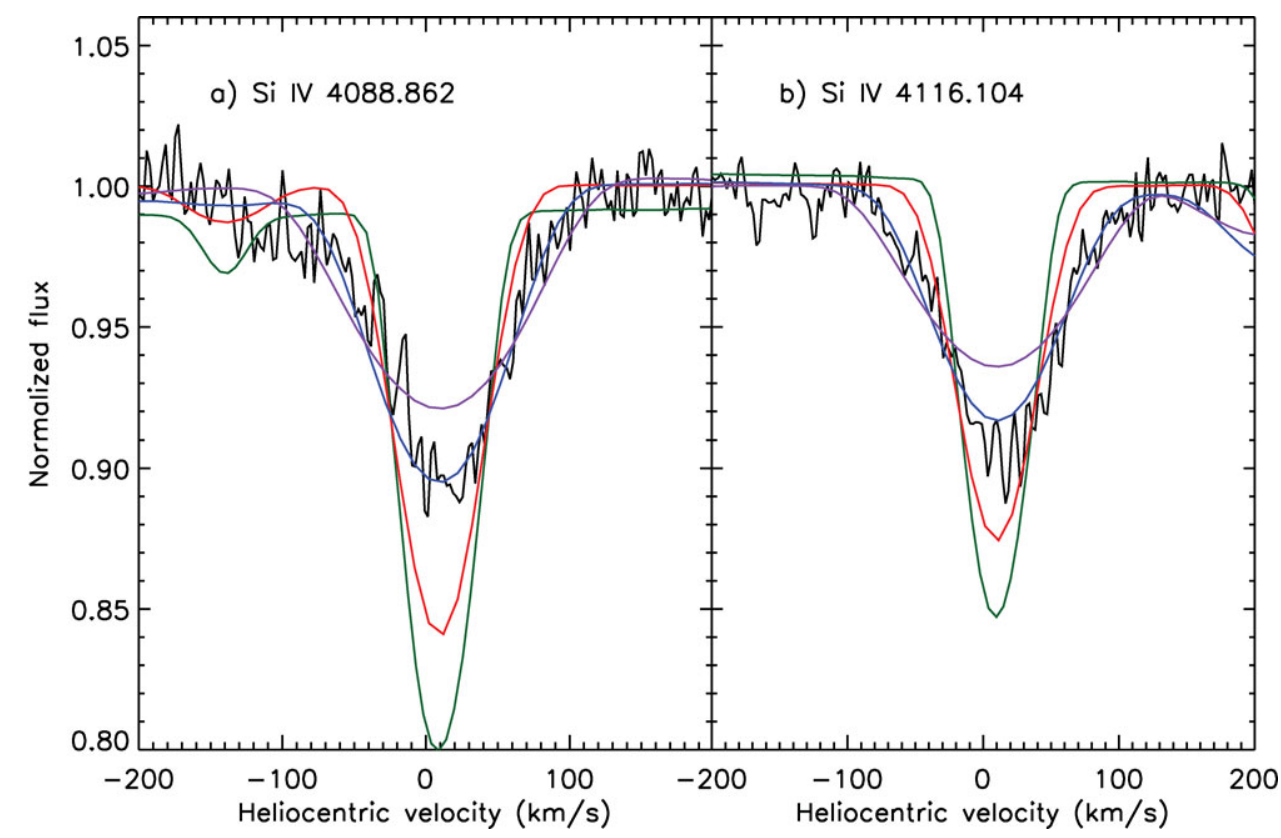

Figure 1. Comparison between the Si IV $\lambda \lambda$ 4088-4116 line profiles of HR Car observed on 2009 May 3rd with synthetic model spectra computed with the 2-D code from Busche \& Hillier (2005) for different rotational velocities, assuming an inclination angle of $i=30^{\circ}: 0 \mathrm{~km} \mathrm{~s}^{-1}$ (green), $75 \mathrm{~km} \mathrm{~s}^{-1}$ (red), $150 \mathrm{~km} \mathrm{~s}^{-1}$ (blue), and $200 \mathrm{~km} \mathrm{~s}^{-1}$ (purple). From Groh et al. (2009a).

Here I discuss the diagnostics that have allowed us to constrain the rotational velocity of LBVs: broadening of spectral lines and latitude-dependent variations of the wind density structure.

\section{Rotational broadening in LBVs}

As in WR stars, the high mass-loss rate of LBVs also cause a ionization stratification of the wind, and spectral lines from ions of different ionization potentials will form at different distances $r$ from the star. The most common lines present in the ultraviolet and optical spectra of LBVs, such as $\mathrm{H} \alpha$ and other Balmer lines, He I, N II, Fe II, and Si III lines, are formed at very large $r$. Since the azimuthal velocity component is proportional to $r^{-1}$, these lines do not show any evidence of rotational broadening, explaining why this phenomenon went undetected for so long.

Lines from high-ionization species, which are formed closer to the stellar photosphere, need to be observed to detect rotational broadening. Thus, in addition to observing LBVs at the right time, one has to look at the right lines. It turns out that Si IV $\lambda \lambda 4088-4116$ lines are the highest ionization lines present in the optical spectrum of most LBVs and, therefore, should be formed closest to the stellar photosphere and are ideal for deriving $v_{\text {rot }}$ in LBVs.

To obtain realistic values of $v_{\text {rot }}$, a 2 -D radiative transfer model which takes into account the dynamical effects of rotation on the wind velocity field are required. We used an updated version of the 2-D code from Busche \& Hillier (2005) to constrain the rotational velocities of the LBVs AG Carinae (Groh et al. 2006) and HR Carinae (Groh et al. 2009a). Figure 1 shows the Si IV 4088 line profiles of HR Car observed during visual minimum (2009 May), compared to synthetic model spectra. Assuming an inclination angle of $i=30^{\circ}$, we derived $v_{\text {crit }} \simeq 170 \pm 20 \mathrm{~km} \mathrm{~s}^{-1}$. 


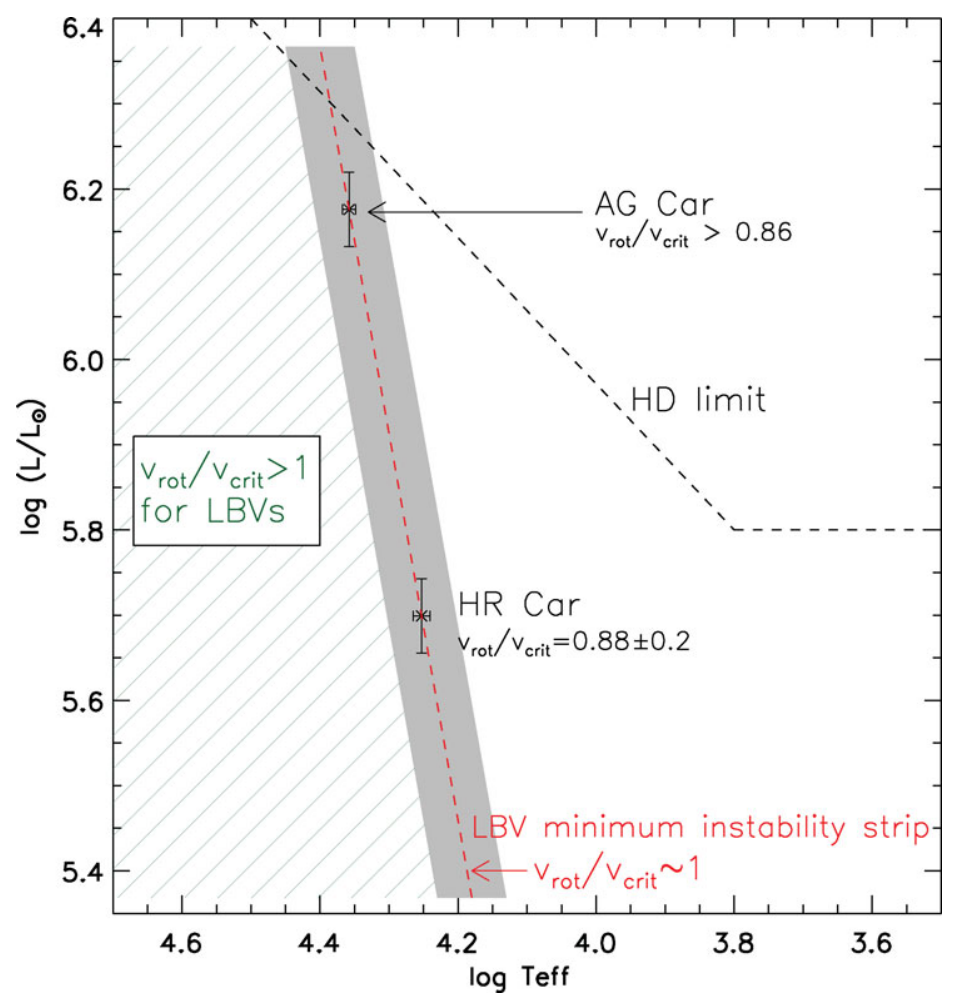

Figure 2. HR diagram showing the position of the LBVs AG Car and HR Car during visual minimum (Groh et al. 2009a,b). The location of the forbidden region for strong-variable LBVs (green hatched region) and the Humphreys-Davidson limit (black dashed line, Humphreys \& Davidson 1994) are shown. From Groh et al. (2009a).

How large is $v_{\text {rot }}$ compared to $v_{\text {crit }}$ for HR Car? Assuming an admittedly uncertain mass of $M=25 M_{\odot}$, our CMFGEN model of HR Car predicts an Eddington parameter of $\Gamma \simeq 0.80$, and we can derive $v_{\text {rot }} / v_{\text {crit }} \simeq 0.88 \pm 0.2$. Therefore, similar to AG Car, for which we found $v_{\text {rot }} / v_{\text {crit }}>0.86$ (Groh et al. 2006), HR Car is also likely rotating very close to, if not at, its critical velocity for break-up at visual minimum.

We have proposed that rotation plays a key role in determining the location of LBVs in the HR diagram during visual minimum. Figure 2 presents the position of AG Car and HR Car in the HR diagram based on their updated stellar parameters (Groh et al. 2009a,b). Previous works have recognized that, during visual minimum, LBVs are located in a welldefined region of the HR diagram, the so-called "LBV minimum instability strip" (Wolf 1989; Clark et al. 2005). Our results suggest that the LBV minimum instability strip is considerably steeper than previously determined, being characterized by $\log \left(L / L_{\odot}\right)=$ $4.54 \log \left(T_{\text {eff }} / \mathrm{K}\right)-13.61$ (red dashed line in Fig. 2). More importantly, we suggest that the LBV minimum instability strip corresponds to the region where critical rotation is reached for LBVs with strong S-Dor type variability. When LBVs are evolving towards maximum, the star moves away from the LBV minimum instability strip (to the right in the HR diagram), and $v_{\text {rot }} / v_{\text {crit }}$ decreases considerably (Groh et al. 2006). The region in the HR diagram on the left side of the LBV minimum instability strip would be populated by unstable LBV stars with $v_{\text {rot }} / v_{\text {crit }}>1$, which would make it a "forbidden region" for LBVs. Indeed, no confirmed strong-variable LBV is seen in this region (van Genderen 2001; Clark et al. 2005). 


\section{Latitude-dependent variations of the wind density structure}

A second way of constraining $v_{\text {rot }}$ in LBVs is by probing the latitudinal dependence of the wind density structure, which is thought to occur when rotation is significant (e.g., Owocki et al. 1996). If a star is surrounded by a reflection nebula with a well-determined 3-D geometry, the latitudinal variation of the wind density can in principle be probed through the reflected stellar spectrum on the nebula. This interesting technique was applied to reflected spectra in the Homunculus nebula around Eta Car, suggesting that the star possesses a faster and denser polar wind (Smith et al. 2003). However, such a configuration is unfortunately not seen in many LBVs, and different techniques need to be employed.

Another neat way of probing latitudinal variations of the wind is by directly resolving the geometry of the wind photosphere, which is most easily achieved in the K-band using near-infrared long-baseline interferometry. This technique was applied to Eta Car using observations gathered with the VINCI (van Boekel et al. 2003) and AMBER (Weigelt et al. 2007) instruments on the Very Large Telescope Interferometer from the European Southern Observatory. The interferometric measurements are consistent with an ellipsoidal shape projected on the sky. Both papers analyzed the measurements using simple geometrical models and interpreted their results as evidence for a dense prolate wind generated by fast rotation, as theoretically predicted by Owocki et al. $(1996,1998)$.

In Groh et al. (2010) we analyzed the published K-band continuum interferometric data of Eta Car, with the goal of constraining $v_{\text {rot }}$ and the spatial orientation of the rotation axis of Eta Car based on the effects of rotation on the wind density structure. This ultimately determines the geometry of the $K$-band emitting region. We computed 2-D latitude-dependent wind models for the primary star (hereafter $\eta_{\mathrm{A}}$ ) in Eta Car using an updated version of the 2-D radiative transfer code of Busche \& Hillier (2005) and via a methodology similar to that described in Groh et al. (2008). The reader is referred to Busche \& Hillier (2005), Groh et al. (2006, 2008, 2009a, 2010), and Driebe et al. (2009) for further details on the code and its applications.

The 2-D code allows for the specification of any arbitrary latitude-dependent variation of the wind density $\rho$ and $v_{\infty}$. For the latitudinal variation of $\rho$, we adopted the predictions from Owocki et al. (1998) for gravity-darkened (GD) line-driven prolate winds,

$$
\frac{\rho(\theta)}{\rho_{0}} \propto \sqrt{1-W^{2} \sin ^{2} \theta},
$$

where $\theta$ is the colatitude angle $\left(0^{\circ}=\right.$ pole, $90^{\circ}=$ equator $), \rho_{0}$ is the density at the pole, and $W$ is the ratio of $v_{\text {rot }}$ to its critical velocity $\left(v_{\text {crit }}\right)$. For consistency, we also assumed the Owocki et al. (1998) predictions for $v_{\infty}(\theta)$. We assumed the same parameters of $\eta_{\mathrm{A}}$ derived by Hillier et al. (2001): stellar temperature $T_{\star}=35,310 \mathrm{~K}$ (at Rosseland optical depth $\left.\tau_{\text {Ross }}=150\right)$, effective temperature $T_{\text {eff }}=9,210 \mathrm{~K}\left(\right.$ at $\left.\tau_{\text {Ross }}=2 / 3\right)$, luminosity $L_{\star}=5 \times 10^{6} L_{\odot}, \dot{M}=10^{-3} M_{\odot} \mathrm{yr}^{-1}$, wind terminal velocity $v_{\infty}=500 \mathrm{~km} \mathrm{~s}^{-1}$, a clumping volume-filling factor $f=0.1$, and distance $d=2.3 \mathrm{kpc}$. The atomic model and abundances are described in Hillier et al. (2001).

We calculated a grid of prolate models with the inclination angle $i$ ranging from $0^{\circ}$ to $90^{\circ}$ in steps of $1^{\circ}$, and $W=v_{\text {rot }} / v_{\text {crit }}$ ranging from 0 to 0.99 in steps of 0.01 . For a desired position angle (PA) orientation on the sky, the $K$-band image, visibilities, and closure phases (CP) were computed. Figure 3 shows the reduced $\chi^{2}$ values of the fit of the model predictions to the VLTI/VINCI visibilities as a function of $W$ and $i$, for prolate models with $\mathrm{PA}=130^{\circ}$. Being conservative, models with $\mathrm{CP} \geqslant 5^{\circ}$ are ruled out, since Weigelt et al. (2007) measured $\mathrm{CP}=0 \pm 2^{\circ}$. Thus, to fit simultaneously the 

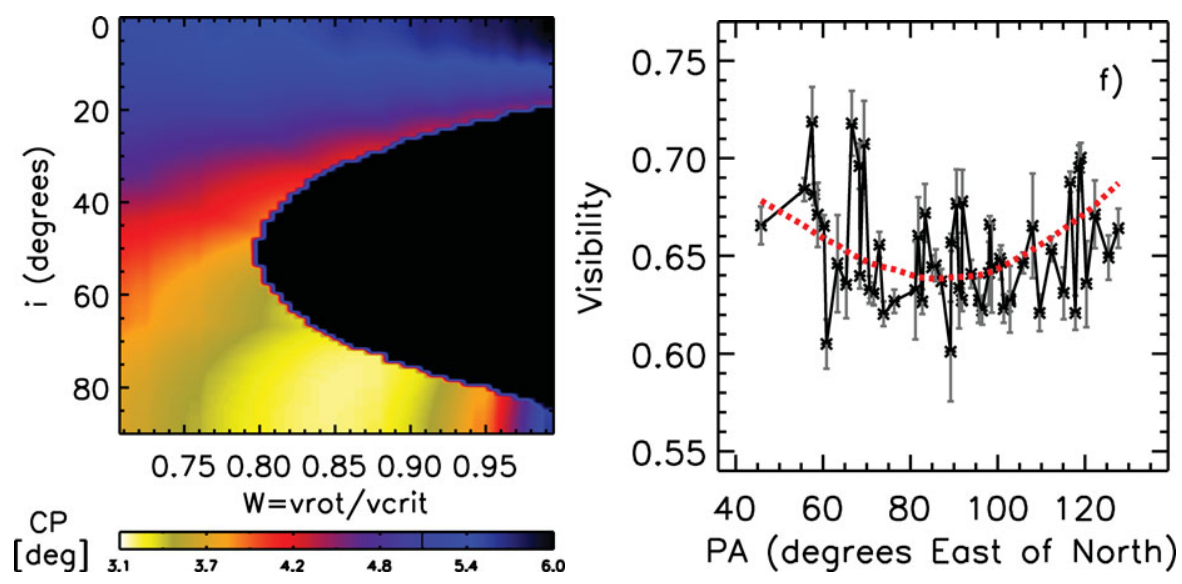

Figure 3. Left panel: $\chi^{2}$ values of the fit of the VINCI visibilities rejecting models with $\mathrm{CP} \geqslant 5^{\circ}$, as a function of $W$ and $i$, for prolate models with PA $=130^{\circ}$ Right: VINCI visibilities for the $24 \mathrm{~m}$ baseline as a function of telescope PA (connected black asterisks) compared to one of the best fit models, with $W=0.92, i=80^{\circ}$, and $\mathrm{PA}=108^{\circ}$ (red dotted line). Note that the projected baseline length of the VINCI measurements changes as a function of PA.

VLTI/VINCI visibilities and the VLTI/AMBER CPs, $W=0.77-0.92$ and $i=60^{\circ}-90^{\circ}$ are needed (Figure 3). Strikingly, this inclination is significantly higher than that of the Homunculus $\left(i_{\mathrm{Hom}}=41^{\circ}\right.$; Smith 2006), which has been assumed so far to align with the current rotation axis of $\eta_{\mathrm{A}}$. We find that, based on the available interferometric data, if $\eta_{\mathrm{A}}$ has a prolate wind, its rotation axis is not necessarily aligned with the Homunculus polar axis.

\section{Short Summary and Future Prospects}

Constraining the rotational velocity of LBVs is crucial for understanding how mass and angular momentum are lost during this short, unstable evolutionary phase, and how that can possibly affect the fate of a massive star. Here we have discussed the two diagnostics that have allowed us to obtain, for the first time, rotational velocities for the most famous and well-observed LBVs such as AG Car, HR Car, and Eta Car. The two diagnostics are complementary, given that rotational broadening requires observations of high-ionization lines which are usually present in the blue region of the optical spectra. Effects of rotation on the wind structure, on the other hand, can be currently probed in the near-infrared, allowing observations of obscured stars.

The comparison between the value of $v_{\text {rot }}$ obtained using these diagnostics might allow us to verify whether the LBVs indeed have denser, faster polar winds as theoretically predicted, and to obtain inclination angles. Indeed, we are analyzing a large dataset of interferometric observations of LBVs in the near-infrared for which the determination of the rotational velocity seems promising.

\section{Acknowledgments}

This paper would not be possible if I had not benefited from a very fruitful collaboration and continuous support from John Hillier. This work is an excellent example of a joint effort by observers and theoreticians that has characterized the massive star field in the last years, and I am more than happy to thank Augusto Damineli, Tom Madura, Stan Owocki, Gerd Weigelt, Rodolfo Barba, Eduardo Fernandez-Lajus, Roberto Gamen, 
Alessandro Moises, Gladys Solivella, and Mairan Teodoro, for sharing their knowledge with me. I also acknowledge financial support from the Max-Planck-Gesellschaft.

\section{References}

Busche, J. R. \& Hillier, D. J. 2005, AJ, 129, 454

Clark, J. S., Larionov, V. M., \& Arkharov, A. 2005, A\& A, 435, 239

Conti, P. S. 1984, in: A. Maeder \& A. Renzini (eds.), Observational Tests of the Stellar Evolution Theory, IAU Symposium 105, p. 233

Driebe, T., Groh, J. H., Hofmann, K.-H., Ohnaka, K. et al. 2009, A\&GA, 507, 301

Groh, J. H., Damineli, A., Hillier, D. J., Barbá, R. et al. 2009a, ApJ (Letters), 705, L25

Groh, J. H., Hillier, D. J., \& Damineli, A. 2006, ApJ (Letters), 638, L33

Groh, J. H., Hillier, D. J., Damineli, A., Whitelock, P. A. et al. 2009b, ApJ, 698, 1698

Groh, J. H., Madura, T. I., Owocki, S. P., Hillier, D. J. et al. 2010, ApJ (Letters), 716, L223

Groh, J. H., Oliveira, A. S., \& Steiner, J. E. 2008, A\&A, 485, 245

Hillier, D. J., Davidson, K., Ishibashi, K., \& Gull, T. 2001, ApJ, 553, 837

Humphreys, R. M. \& Davidson, K. 1994, PASP, 106, 1025

Maeder, A. \& Meynet, G. 1994, $A \mathscr{E} A, 287,803$

Maeder, A. \& Meynet, G. 2000a, A\&SA, 361, 159

Maeder, A. \& Meynet, G. 2000b, ARAA, 38, 143

Meynet, G. \& Maeder, A. 2003, A\&A, 404, 975

Owocki, S. P., Cranmer, S. R., \& Gayley, K. G. 1996, ApJ (Letters), 472, L115

Owocki, S. P., Cranmer, S. R., \& Gayley, K. G. 1998, Ap\&SS, 260, 149

Smith, N. 2006, ApJ, 644, 1151

Smith, N., Davidson, K., Gull, T. R., Ishibashi, K. et al. 2003, ApJ, 586, 432

van Boekel, R., Kervella, P., Schöller, M., Herbst, T. et al. 2003, A\&A, 410, L37

van Genderen, A. M. 2001, A\&A, 366, 508

Weigelt, G., Kraus, S., Driebe, T., Petrov, R. G. et al. 2007, A\&AA, 464, 87

Wolf, B. 1989, $A \mathscr{E} A, 217,87$

\section{Discussion}

MEYNET: I have a question regarding the HR diagram where the forbidden region is in the left of the LBV minimum instability strip. I would imagine that the star evolves from the blue to red, encounters the instability, suffers strong mass loss which brings the stars to the right because of the optical thickness of the wind.

GroH: The optical thickness of the wind is included in our calculations for the positions of the LBVs in the HR diagram. Apparently, the stars are very close to the Eddington limit modified by rotation at the LBV minimum instability strip, but in the case of AG Car and HR Car we do not see a runaway increase in mass loss, which is actually comparable to other LBVs. That is related to the very important issue of how much rotation increases the mass-loss rate and certainly deserves future studies.

Puls: 1. You referred to the critical velocity for break-up. Since you are dealing with LBVs ( $\Gamma$ close to unity), did you include the effects of the Eddington limit modified by rotation $(\Omega \Gamma$ limit)? 2. Which kind of models are underlying your analysis?

Gron: First, yes, we include the effects of rotation on the Eddington limit following Maeder \& Meynet (2000a). In these analyses we have used an updated version of the 2-D code from Busche \& Hillier (2005) using a prescribed latitudinal variation of the wind density as a function of $v_{\text {rot }}$ as in Owocki et al. (1996). 